\title{
Treatment of Localized Morphea with Zyderm Collagen Implant
}

JOHN G. STONER, M.D. NEIL A. SWANSON, M.D. RONALD J. SIEGLE, M.D.

Zyderm collagen implant ( $\mathrm{ZCl})$, used in the treatment of scarring, soft tissue contour defects, and age lines, has been found to be both efficacious and safe. Over 40,000 individuals have received ZCI, with fewer than $2 \%$ showing adverse responses (data on file, Collagen Corporation, Palo Alto, California). $\mathrm{ZCl}$ is comprised of highly purified bovine collagen that has undergone partial enzymatic degradation to reduce its antigenicity. ${ }^{1}$ Despite this purification, theoretical considerations have precluded its use in patients with autoimmune diseases, such as lupus erythematosus, inflammatory bowel disease, Hashimoto's thyroiditis, and progressive systemic sclerosis. Morphea has not been considered on this list of contraindicated diseases. It is usually an isolated phenomenon with no predisposition to systemic disease. Our patient represents the first case of ZCI used to treat cosmetically disfiguring localized morphea. ZCI injections were instituted following consultation and consent from the patient and representatives of the Collagen Corporation.

John G. Stoner, M.D., is Clinical Assistant Professor, Department of Dermatology, Johns Hopkins University, Baltimore, Maryland.

Neil A. Swanson, M.D., is Assistant Professor of Dermatology and Otorhinolaryngology-Head and Neck Surgery, University of Michigan, Ann Arbor, Michigan.

Ronald J. Siegle, M.D., is Assistant Professor of Medicine, Division of Dermatology, The Ohio State University, Columbus, Ohio.

Address reprint requests to Neil A. Swanson, M.D., Department of Dermatology, University of Michigan, C2065 Outpatient Building, Ann Arbor, MI 43109.

626

\section{CASE REPORT}

The patient is a 25-year-old woman with a 4-year history of a sclerotic, atrophic, and somewhat hyperpigmented $2.0 \times 3.5 \mathrm{~cm}$ patch over her left lower eyelid, zygoma, and cheek (Fig. 1). Historically the lesion had not increased in size for 2 years and was thought to be clinically inactive. The patient had no signs or symptoms of concomitant connective tissue disease, and the physical examination was normal. A biopsy of the lesion was consistent with morphea. Antinuclear antibody (ANA) and complete blood count were normal. ZCI test dose injected into her right volar forearm was nonreactive at 72 hours and at 1 month. At monthly intervals over the following 3 months, a total of $1.5 \mathrm{ml} \mathrm{ZCI}$ was implanted into the lesion up to, but not extending over, the orbital rim. The patient achieved a very good clinical correction $(80 \%)$ and was entirely satisfied with the cosmetic results (Fig. 2). This result has persisted for 1 year, and careful followup for loss of correction is anticipated. Prior to, during, and following ZCI therapy, anti-ZCI antibodies assays remained normal. The antibodies were measured in our laboratory using an ELISA assay developed and reported by Swanson et al. ${ }^{2}$ In this assay, the patient's serum has remained well within normal limits for our laboratory.

\section{COMMENTS}

For this patient, ZCI was able to correct the contour defect caused by her disease. However, the implant had no effect on the hyperpigmentation on the sclerotic texture of the lesion. The latter is in contrast to acne scarring, where $\mathrm{ZCI}$ often softens the existing scar (data on file, Collagen Corporation).

\footnotetext{
J. Dermatol. Surg. Oncol. 10:8 August 1984
} 


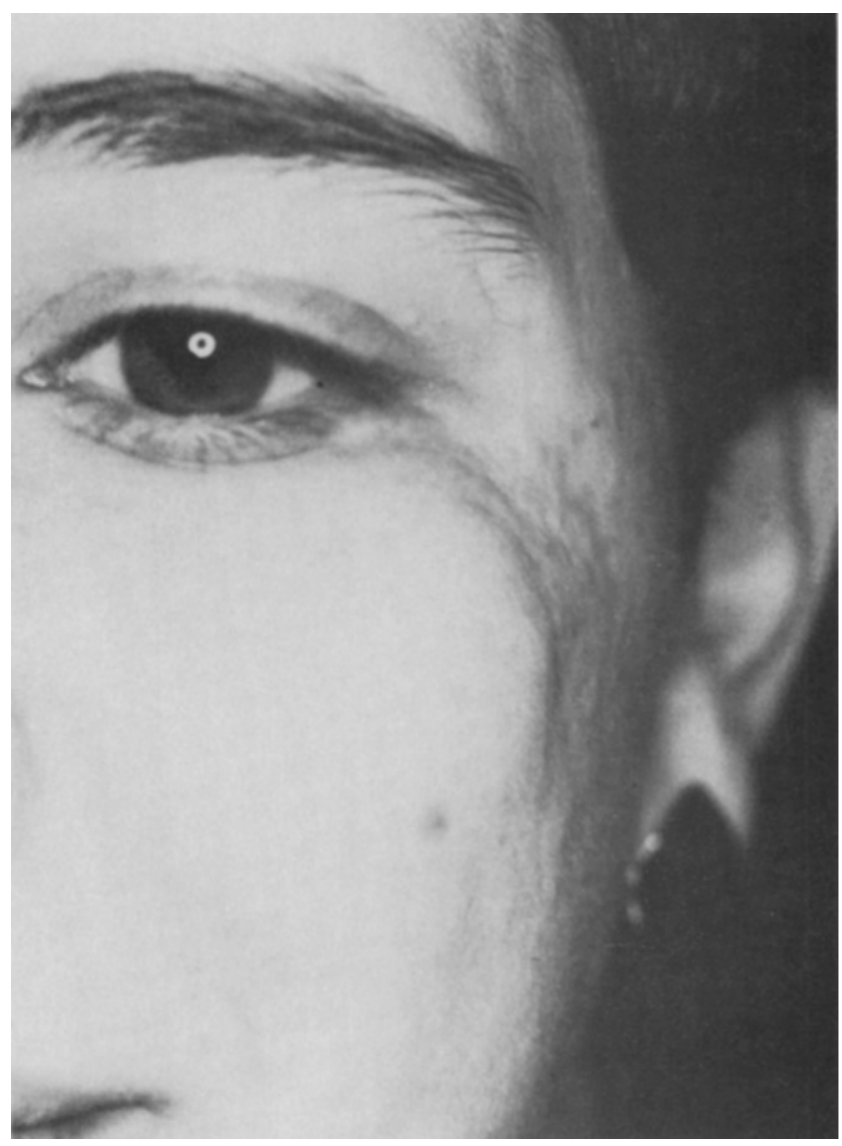

FIGURE 1. Pre-Zyderm implant.

The presence of autoimmune connective tissue disease (CTD) is an absolute contraindication to the administration of ZCI. The diagnosis of one of these diseases is made through history, serologic testing, $x$-rays, and other diagnostic procedures. Patients with the variant en Coup de Sabre of linear scleroderma have frequently been found to have positive ANA as well as other signs and symptoms of CTD. ${ }^{3,4}$ Our patient, by history, physical examination, and laboratory findings, did not fit this subset of scleroderma. Her disease was inactive, her physical examination was entirely normal, and her ANA was negative. For these reasons, we thought she had localized morphea only, with no predisposition to systemic disease. Serologic testing for anti-ZCI antibodies was also negative. To date, this highly specific test for anti-ZCI antibodies had been found positive in about $7 \%$ of the control population and $100 \%$ of patients who have received $\mathrm{ZCI}$ and exhibited a positive skin test or treatment site reaction. ${ }^{5}$ It is entirely possible that this assay can be used to screen patients at risk to develop adverse $\mathrm{ZCI}$ reactions. In doing so, the list of contraindicated diseases may be modified to allow more patients to receive $\mathrm{ZCI}$.

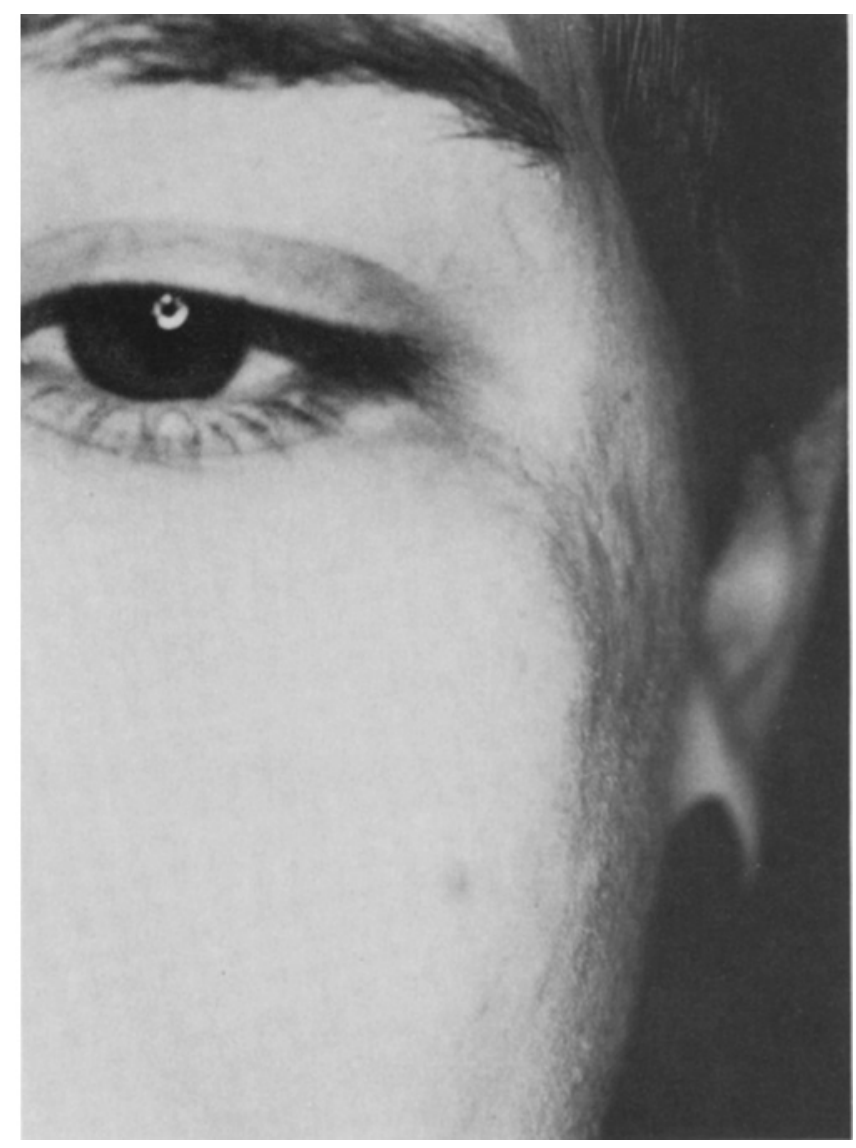

FIGURE 2. A good correction (about $80 \%$ ) following the injection of $1.5 \mathrm{ml}$ of Zyderm.

In summary, we present a new indication for $\mathrm{ZCI}$. Although skin hyperpigmentation was not affected by the implants, the contour depression was elevated sufficiently to achieve satisfactory cosmesis. This correction has persisted to date, although we anticipate the need for reinjection to maintain correction in the future. Finally, anti-ZCI antibodies were negative, suggesting that the risk of an adverse response was minimal.

\section{REFERENCES}

1. Knapp, T. F., Luck, E., and Daniels, J. F. Behavior of solubilized collagen as a bioimplant. J. Surg. Res. 23:96-105, 1977.

2. Swanson, N. A., Stoner, J. G., Siegle, R. J., and Solomon, A. R. Treatment site reactions to Zyderm collagen implanation. J. Dermatol. Surg. Oncol. 9:377-380, 1983.

3. Mackel, S. E., Kozin, F., et al. Concurrent linear scleroderma and systemic lupus erythematosus: A report of two cases: J. Invest. Dermatol. 73:368-372, 1979.

4. Mitchell, A. J., Rusin, L. J., and Diaz, L. A. Circumscribed scleroderma with immunologic evidence of systemic lupus erythematosus: Report of a case and review of the literature. Arch. Dermatol. 116:69-73, 1980.

5. Siegle, R. J., McCoy, P. J., Schade, W., and Swanson, N. A. Humoral immune responses associated with clinical reactions following intradermal implantation of bovine collagen. Arch. Dermatol., in press 